\title{
Estimation of Genetic Correlations and Selection Responses for Carcass Traits between Ultrasound and Real Carcass Measurements in Hanwoo Cows
}

\author{
Jihyun Son and Deukhwan Lee* \\ Department of Animal Life Resources, Hankyong National University, Anseong 456-749, Republic of Korea
}

\begin{abstract}
This study was conducted to determine genetic correlations among carcass traits measured by ultrasound and real carcass measurements and to estimate indirect selection responses for real carcass traits based on ultrasound measurements in Hanwoo cows. To accomplish this, 22,080 ultrasound measurement records from 17,926 cows collected from 2001 to 2012 and 11,907 carcass records obtained from fattened cattle from 2008 to 2012 were used. Genetic parameters were estimated based on eye muscle area (EMA), backfat thickness (BF) and marbling score (MS) measured by ultrasound-scanning of live cows and using the official technique on chilled bovine half-carcasses after slaughtering.

Heritability and genetic correlation for carcass traits were estimated using a mixed model equation that consisted of environmental effects as fixed parameters and additive genetic effects and residual effects as random parameters, assuming that traits were different between ultrasound and carcass measurements. This statistical method was applied to the average information restricted maximum likelihood method. The heritability of EMA, BF and MS measured by ultrasound were 0.33 , 0.61 and 0.46 , respectively, while the heritability estimates of the corresponding traits based on carcass measurements were 0.29 , 0.40 and 0.38 , respectively and the genetic correlation between ultrasound and carcass traits for EMA, BF and MS were $0.41,0.78$ and 0.67 , respectively. The genetic correlation between ultrasound and carcass traits was highly positive. Additionally, the selection response for marbling score was estimated to be 0.42 per generation if the cows were selected based on the ultrasound scan marbling score with an assumed selection intensity of 0.8 . Overall, these results indicate that the ultrasound scan technique would be applicable to judging cow selection for genetically improved meat quality.
\end{abstract}

(Key words : Hanwoo, Carcass trait, Ultrasound measurement, Heritability, Genetic correlation, Indirect selection, Selection response)

\section{INTRODUCTION}

Carcass traits related to the quality of Hanwoo (Korean indigenous beef cattle) meat are major traits that greatly affect the income level of farmers (Kim et al., 2010; Cho, 2013). Since marbling score and backfat thickness are indicators of meat quality that are highly heritable (Yoon et al., 2002; Rho, 2002; Choi et al., 2006), many Korean farmers that raise Hanwoo cows prefer the semen of bulls that are genetically superior with regard to these traits. Progeny tests to select proven bulls with excellent genetic merits at the national level were first conducted in 1982. Since then, around 40 proven bulls have been selected every year. From the first half of 2008, meat quality has been judged based on weights of $1,-1$, or 6 , which are assigned depending on the weight of the carcass, backfat thickness and marbling score during calculation of comprehensive selection indices for Hanwoo bulls (National Institute of Animal
Science, 2008; Park et al., 2010). The genetic improvement of Hanwoo cows began in 1979 with the establishment of regional Hanwoo cow complexes. By early 2000, the basic data describing genetic improvement of Hanwoo cows and calf production had been secured and a base was prepared for Hanwoo improvement by the establishment of these regional complexes. Since the 2000s, it has been possible to measure slaughter-related traits such as backfat thickness, longissimus muscle area and marbling score, which are related to meat quality, by using ultrasonic measurement equipment as an indirect method in live cattle, which could otherwise only be measured after slaughtering the cows. Thereafter, the National Agricultural Cooperatives Federation and some Hanwoo breeding farms conducted meat quality inspection using an ultrasonic measurement technique. This ultrasonic measurement technique has been used to decide the outgoing time of fattening cattle. In addition, regional Hanwoo complexes were established uniting Hanwoo farms

* Corresponding author: Deukhwan Lee, Department of Animal Life Resources, Hankyong National University, Chungang-no Anseong-si Gyeonggi-do, Republic of Korea 456-749. Tel: 031-670-5091, Fax: 031-676-5091, E-mail: dhlee@hknu.ac.kr 
Son et al.; Genetic Correlations and Selection Responses for Carcass Traits between Ultrasound and Real Carcass Measurement

in cities and districts nationwide, and the ultrasonic measurement technique has been actively utilized as a means of breeding cow improvement at the regional level since 2010 (Lee, 2003; Roh et al., 2010). Some research has investigated the correlation of meat quality related traits between methods using ultrasound equipment and actual slaughtered carcasses (Lee et al., 2006; Koo, 2007; Lee et al., 2008; Yoon, 2010; Lee et al., 2011; Kim et al., 2012). However, research concerning the genetic relationships between these traits is still needed, as is efficient improvement of Hanwoo by setting a breeding strategy.

The present study was conducted to investigate the efficacy of ultrasound scanning of live Hanwoo cows for genetic improvement of their genetic potentials based on carcass traits. Here, we examined genetic correlation among traits to verify the reliability of data for meat quality improvement of Hanwoo by investigating carcass characteristics that have pedigree information through ultrasonic measurement data.

\section{MATERIALS AND METHODS}

\section{Ultrasonic and carcass data}

The ultrasound measurement data for meat quality related traits used in the present study were collected from Hanwoo cows at least 15 months in age from 1,021 beef cattle farms throughout South Korea from 2001 to 2012. The data from the carcasses of fattened cattle that have genetic relationships with ultrasonic measured cows were collected by publicly certified meat quality judges from 1,054 beef cattle farms from 2008 to 2012. Records with greater than 3 standard deviations were excluded from the study to remove outliers. Therefore, a total of 22,080 ultrasound measurement data were gathered from 17,926 cows and a total of 11,907 carcass data were acquired from 8,747 cows, 203 bulls and 2,957 steers for genetic analysis. The number of cows for which repeated ultrasound measurements were carried out was 3,485 , and both ultrasound measurements and carcass data were obtained from 384 cows. The carcass traits considered in the present study were eye muscle area (EMA), backfat thickness (BF) and marbling score (MS), which have important economic values among the characteristics graded by the Korean Institute for Animal Products Quality Evaluation. The traits measured by ultrasound were eye muscle area (UEMA), backfat thickness (UBF) and marbling score (UMS). Traits were measured so that the shape of the eye muscle area could be examined between the $13^{\text {th }}$ spine and the first lumber vertebra, which is the same location used for the carcass grading system. For ultrasound image measurement, a Real-time B mode SV900 and Pico from MEDISON with an attached $3.5 \mathrm{MHz}$ Leaner probe was used. UEMA and UBF were calculated by a devoted ultrasound image analysis program and UMS wasmarked as a score in a range of 1-27 based on the subjective judgment of experts.

\section{Statistical methods}

Investigated ultrasound traits showed different growth modes and non-linear growth patterns according to the age in months of the cows (Fig. 1). Thus, the data were corrected for each trait using the Hanwoo cow standard growth curve (Lee et al., 2008), which estimates the growth curve of cows according to their age in months using a function proposed by von Bertalanffy (Bertalanffy, 1957). Finally, a statistical model was set up for genetic analysis.

Since the fixed effects affected by the traits differed between ultrasound traits and carcass traits, environmental factors that influence observation were evaluated by analysis of variance (ANOVA) using the GLM procedure of the SAS statistical program (package ver. 9.2). These environmental factors showed a high degree of difference among all considered traits. Thus, a linear model equation for the ultrasound measurement traits and carcass traits was established:

$$
Y_{i j k}=\mu_{i}+B C S_{i j}+H Y M_{i k}+\beta_{1} C G_{i j k}+a_{i j k}+e_{i j k}
$$

For the above equation, it was assumed that $\mathrm{Y}_{\mathrm{ijk}}$ is the observation measured within the $j^{\text {th }}$ BCS and the $k^{\text {th }}$ HYM effect of the $i^{\text {th }}$ trait, $\mathrm{BCS}_{\mathrm{ij}}$ is the fixing effect of body condition score (BCS), $\mathrm{HYM}_{\mathrm{ik}}$ is the random effect of farm-year-month, $\mathrm{CG}_{\mathrm{ijk}}$ is the observed chest girth of each individual cow, $\beta_{1}$ is the covariate of chest girth, $a_{i j k}$ is the random additive genetic effect of a cow and $\mathrm{e}_{\mathrm{ijk}}$ is the random residual effect. The multi-trait mixed model for the carcass measurement traits is as follows:

$$
\begin{aligned}
Y_{i j k l m n}= & \mu_{i}+\operatorname{Sex}_{i j}+\operatorname{Herd}_{i k}+\operatorname{SYS}_{i l}+B_{i m}+\beta_{2} \text { day }_{\mathrm{ijklmn}}+a_{i j k l m n}+ \\
& \mathrm{e}_{\mathrm{ijklmn}}
\end{aligned}
$$

where $Y_{i j k l m n}$ is the observation of the $n^{\text {th }}$ slaughtered cow of $\mathrm{j}^{\text {th }} \operatorname{sex}\left(\mathrm{Sex}_{\mathrm{ij}}\right.$ - cow, bull, steer) from the $\mathrm{m}^{\text {th }}$ slaughter house $\left(\mathrm{B}_{\mathrm{im}}\right)$ during the $1^{\text {th }}$ slaughter year-season $\left(\mathrm{SYS}_{\mathrm{il}}\right)$ having the $i^{\text {th }}$ trait. Sex $_{i j}$ is the gender effect as a fixed effect, Herd ${ }_{i k}$ is the effect of a beef cattle farm, SYS il is the effect of 


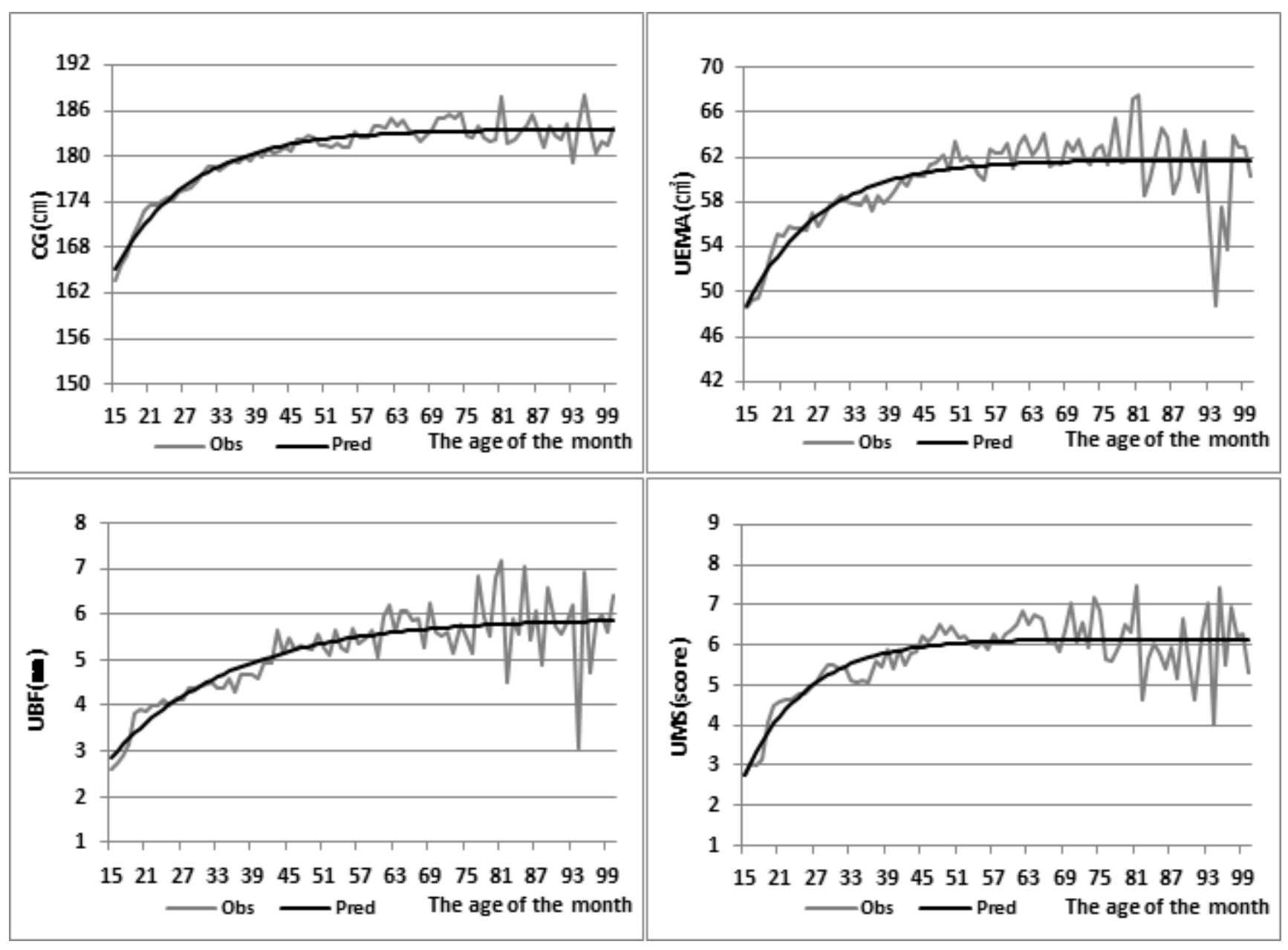

Fig.1. Estimated growth curves for chest girth(CG), eye muscle area(UEMA), backfat thickness(UBF) and marbling scores(UMS) by months of age in Hanwoo cows.

slaughter year-season, $B_{i m}$ is the slaughter house effect, day $y_{i j k l m n}$ is the observed value on the age (month) effect at slaughtering, $\beta_{2}$ is the coefficient of covariate for day $\mathrm{ijk}_{\mathrm{j} k \mathrm{mn}}, \mathrm{a}_{\mathrm{ijklmn}}$ is the random additive genetic effect and $\mathrm{e}_{\mathrm{ijk} k \mathrm{mn}}$ is the random residual effect.

\section{Estimation of variance components}

A normal equation was established by mixing the above two linear mixed models. The null hypothesis was set in the normal equation as below:

$\operatorname{Var}\left[\begin{array}{c}a_{U E M A} \\ a_{U B F} \\ a_{U M S} \\ a_{E M A} \\ a_{B F} \\ a_{M S}\end{array}\right]=G_{0} \otimes A$, where $G_{o}$ is the genetic (co) variance matrix for the six traits, A is the animal genetic relationship matrix between animals and $\otimes$ is the Kronecker product.

The (co) variance components for the random herd-year-month of ultrasound measurement traits were assumed as follows:

$\operatorname{Var}\left[\begin{array}{c}H Y M_{U E M A} \\ H Y M_{U B F} \\ H Y M_{U M S}\end{array}\right]=H_{0} \otimes I_{H Y M}$,

where $H_{o}$ is the (co) variance matrix for the three traits measured by ultrasound, $\mathrm{I}_{H Y M}$ is the unit matrix having a size of the HYM level and $\otimes$ is the Kronecker product.

The described model was employed to estimate the heritability of each trait and the genetic relationships between traits was calculated using Linux based AIREMLF90 (Misztal and Tsuruta, 2002). 


\section{RESULTS AND DISCUSSION}

\section{General performance}

Basic statistical analysis was carried out for all records of traits measured by ultrasound in cows from approximately 15 to 150 months of age with an average age of 38 months. The average eye muscle area and backfat thickness were around $58 \mathrm{~cm}^{2}$ and $4.59 \mathrm{~mm}$, respectively. The average marbling score $(1=$ lowest, $27=$ highest $)$, which represents fat content in muscle, was 5.32. Since the traits related to meat quality can change with the age of the cattle, the observations of these traits on each individual were transformed the deviates from a non-linear growth curve by month of age for corresponding trait proposed by Lee et al.(2008).

The carcass traits were measured for cows, steers and fattened bulls of around 20 to 145 months of age with an average age of approximately 55. The average eye muscle area was around $83 \mathrm{~cm}^{2}$, the average backfat thickness was around $12.42 \mathrm{~mm}$ and the average marbling score was around 4.41. As shown in Table 1, the traits differed significantly, even when corrected by growth curve function $\left(\mathrm{EMA}=58: 83 \mathrm{~cm}^{2}, \mathrm{BF}=\right.$
$4.59: 12.42 \mathrm{~mm}$, score converted by 9 points, $\mathrm{MS}=1.77: 4.41$ point). This difference might have been due to variations in the feeding management of cows and fattened cattle.

The pedigree construction of the data used in the present study was based on 17,926 cows fathered by 243 sires and the meat quality traits of 22,000 records measured by ultrasound equipment and chest girth data measured at the same time. However, the carcass data obtained from 11,907 cattle were comprised of around 8,747 cows, 2,957 steers and fattened bulls born from 93 sires.

Before setting up the statistical model for genetic evaluation, ANOVA of ultrasound measured carcass traits and real carcass traits was performed. Statistical models for analysis of variance considered body condition score (BCS), herd-yearmonth as the class classification and chest girth as the covariate for the ultrasound measured carcass traits, as well as sex, herd, slaughter year-season, and slaughter house as the class classification and days of age at slaughter for the actual carcass traits. All effects on the models significantly influence to the considered traits (Table 3 and 4). Therefore, all effects were considered in the statistical mixed model for estimation of the genetic parameters.

Table 1. Simple statistics of carcass traits measured by ultrasound-scan and observation of real carcasses in Hanwoo cows

\begin{tabular}{|c|c|c|c|c|c|}
\hline Traits (unit) & Mean & SD & $\mathrm{CV}$ & Min & Max \\
\hline \multicolumn{6}{|l|}{ Ultrasound scan $(\mathrm{N}=22,080)$} \\
\hline Age at Measuring (months) & 37.84 & 17.02 & 44.97 & 15.01 & 149.52 \\
\hline CG (cm) & 177.86 & 8.74 & 4.92 & 145.00 & 220.00 \\
\hline UEMA $\left(\mathrm{cm}^{2}\right)$ & 58.03 & 11.28 & 19.45 & 16.84 & 107.40 \\
\hline UBF (mm) & 4.59 & 3.05 & 66.60 & 1.00 & 20.00 \\
\hline UMS (score) & 5.32 & 3.25 & 61.15 & 1.00 & 26.00 \\
\hline \multicolumn{6}{|l|}{ Real carcass $(\mathrm{N}=11,907)$} \\
\hline Age at Measuring (months) & 54.50 & 21.81 & 40.02 & 19.70 & 144.83 \\
\hline CW (kg) & 356.47 & 56.41 & 15.83 & 182.00 & 581.00 \\
\hline EMA $\left(\mathrm{cm}^{2}\right)$ & 83.06 & 10.94 & 13.18 & 31.00 & 160.00 \\
\hline $\mathrm{BF}(\mathrm{mm})$ & 12.42 & 5.44 & 43.83 & 2.00 & 40.00 \\
\hline MS (score) & 4.41 & 2.10 & 47.62 & 1.00 & 9.00 \\
\hline
\end{tabular}

Abbreviations: CW, carcass weight; EMA, eye muscle area of carcass; BF, backfat thickness of carcass; MS, marbling score of carcass; UEMA, ultrasound-scanned eye muscle area; UBF, ultrasound-scanned backfat thickness; UMS, ultrasound-scanned marbling score

Table 2. Pedigree information and observed records of ultrasound-scan and real carcass data in Hanwoo cows

\begin{tabular}{lrcrrrc}
\hline & Sire & No. progeny/Sire & Cow & Steer & Bull & No. Record \\
\hline \hline Ultrasound-scan & 243 & 90.9 & 17,926 & 0 & 0 & 22,080 \\
Real carcass & 93 & 128.0 & 8,747 & 2,957 & 203 & 11,907 \\
\hline
\end{tabular}


Son et al.; Genetic Correlations and Selection Responses for Carcass Traits between Ultrasound and Real Carcass Measurement

Table 3. Mean squares and tests of significance for ultrasonic measurement of carcass traits based on fixed effects on a statistical model

\begin{tabular}{lrccc}
\hline Source & \multicolumn{1}{c}{ df } & UEMA & UBF & UMS \\
\hline \hline BCS & 7 & $4267.92^{* * *}$ & $662.44^{* * *}$ & $224.77^{* * *}$ \\
HYM & 1341 & $669.24^{* * *}$ & $47.25^{* * *}$ & $56.42^{* * *}$ \\
$\beta_{1}$ & 1 & $102127.47^{* * *}$ & $2501.71^{* * *}$ & $4431.41^{* * *}$ \\
error & 20730 & 48.33 & 4.43 & 5.37 \\
\hline
\end{tabular}

Abbreviations: UEMA, ultrasound-scanned eye muscle area; UBF, ultrasound-scanned backfat thickness; UMS, ultrasound-scanned marbling score; BCS, fixing effect of body condition score; HYM, random effect of farm-year-month; CG, observation for chest girth of each individual cow; $\beta_{1}$, covariate of chest girth; Significance: $\mathrm{p}<0.0001=* * * ; \mathrm{p}<0.01=* * ; \mathrm{p}<0.05=* ; \mathrm{p}>0.05=\mathrm{ns}$.

Table 4. Mean squares and tests of significance for real carcass traits based on fixed effects on a statistical model

\begin{tabular}{lrrrr}
\hline Source & \multicolumn{1}{c}{ df } & EMA & \multicolumn{1}{c}{ BF } & \multicolumn{1}{c}{ MS } \\
\hline \hline Sex & 2 & $66,110.84^{* * *}$ & $3,264.08^{* * *}$ & $1,825.35^{* * *}$ \\
Herd & 1053 & $302.97^{* * *}$ & $49.48^{* * *}$ & $7.33^{* * *}$ \\
SYS & 19 & $916.4^{* * *}$ & $98.27^{* * *}$ & $19.38^{* * *}$ \\
B & 54 & $610.4^{* * *}$ & $82.42^{* * *}$ & $18.72^{* * *}$ \\
$\beta_{2}$ & 1 & $110.58^{\text {ns }}$ & $6,716.29^{* * *}$ & $16.53^{* *}$ \\
error & 11777 & 85.76 & 24.92 & 3.20 \\
\hline
\end{tabular}

Abbreviations: EMA, eye muscle area on carcass; BF, backfat thickness on carcass; MS, marbling score on carcass; Sex, gender effect as a fixed effect; Herd, effect of a beef cattle farm; SYS, effect of slaughter year-season; B, slaughter house effect; $\beta_{2}$, coefficient of covariate for day $\mathrm{ijklmn}_{\mathrm{j}}$; Significance: $\mathrm{p}<0.0001=* * * ; \mathrm{p}<0.01=* * ; \mathrm{p}<0.05=* ; \mathrm{p}>0.05=\mathrm{ns}$.

\section{Heritability}

Estimations of heritability, genetic correlation and phenotypic correlation for the carcass traits (CT) and ultrasound traits (UT) calculated by the (co)variance components of each trait obtained using AI (Average Information)(Gilmour et al., 1995) are provided in Table 5.

The heritability of the carcass trait of eye muscle area was estimated to be 0.29 in this study. This value was lower than that of the heritability estimate (0.45) for the EMA of Angus fattened bulls reported by Kemp et al. (2002) or the heritability estimate (0.35) for the EMA of Japanese Brown Cattle proposed by Hirooka et al. (1996). However, it was similar to the EMA heritability estimates of $0.27,0.35$ and 0.33 in the same breed (Hanwoo) reported by Yoon et al. (2002), Roh (2004) and Choi et al. (2006), respectively. Moreover, the eye muscle area (UEMA) carcass trait measured by ultrasound in the present study was 0.33 , which was similar to that of EMA. The heritability estimate of UEMA for the beef cattle was similar to the values of 0.31 and 0.29 for beef cattle proposed by Stelzleni et al. (2002) and Kemp et al. (2002). However, it was somewhat lower than the heritability of 0.45 estimated from crossed beef cattle steers proposed by Devitt et al. (2001) or the heritability estimate of 0.49 for beef cattle heifers reported by Crews et al. (2001). The UEMA heritability of carcass traits measured by ultrasound for Hanwoo cows reported to date include 0.17 (Lee and Kim, 2004), 0.11 (Lee et al., 2006), 0.15 (Koo, 2007) and 0.15 (Yoon, 2010), implying that the heritability of eye muscle area estimates in the present study was relatively high.

The heritabilities of $\mathrm{BF}$ and UBF for the carcass traits in the present study were estimated to be 0.40 and 0.61 , respectively. Utrera et al. (2004) reviewed 61 theses that investigated the heritability of beef cattle and reported an average heritability of BF 0.40 . Heritability values similar to those found in the present study of $0.35,0.39$ and 0.38 were reported for Hanwoo cows by Yoon et al. (2002), Roh (2004) and Lee and Kim (2004), respectively. The UBF of Hanwoo cows has generally been reported to have low heritability, though it differs from the composition of the subject population (Lee et al., 2006; Koo, 2007; Lee et al., 2011). Yoon (2010) estimated the genetic parameters of ultrasound traits using a similar design and correction method as the present study. Specifically, he corrected for the age and body condition score of the ultrasound records 
collected from around 20,000 Hanwoo cows in ten areas from all over South Korea using anon-linear mixed model equation. He then proposed a relatively higher UBF heritability of 0.39 through a multi-trait mixed model equation. Stelzleni et al. (2002) estimated the heritability of the BF trait for Brangus bulls and cows as 0.26 , which differed greatly from the UBF observed in the present study. However, Crew et al. (2003) and Reverter et al. (2002) estimated UBF heritability values for Simmental heifers and Australian Angus of 0.69 and 0.54 , respectively, which was similar to the heritability measured in the present study.

Marbling score is a very important economic meat quality. It has been reported that, although marbling maturity is completed by around five years in cows, variation among cows is considerably high (Lee et al., 2008). In the present study, the MS of the carcasses and the marbling score of the ultrasound trait(UMS) were estimated to be 0.38 and 0.46 , respectively, which are similar to the MS heritability of BF 0.37 reported by Utrera (2004). Most studies conducted to date have provided MS heritability estimates of greater than 0.37 for beef cattle and Hanwoo (Kemp et al., 2002; Hirooka et al., 1996; Devitt et al., 1996; Crews et al., 2003; Yoon et al., 2002; Roh, 2004 Lee and Kim, 2004; Choi, 2006). The heritability estimates of UMS by Crews et al. (2003) and Reverter et al. (2000) were 0.54 and 0.43 for Simmental heifers and Australian Angus, respectively, which were similar to the results of the present study. Yoon (2010) proposed a relatively high heritability estimate of 0.38 for the trait of marbling score by ultrasound for Hanwoo cows. However, most UMS heritability values from Hanwoo cows in previously conducted studies were less than 0.24 (Lee et al., 2006; Koo, 2007; Lee et al., 2011).

The discrepancies among studies could be due to the use of different breeds and varying population compositions. Estimates of UEMA, UBF and UMS heritability were high when compared to the existing research. This may have been because of increased genetic variation between subjects owing to the size of the population used in the present study being larger and the data being collected over a longer duration. The heritability of ultrasound traits estimated in the present study was similar to those from carcass traits with relatively higher variations. Furthermore, as Lee et al. (2008) reported, since variation is generated between subjects with different ages according to growth level, each ultrasound trait was corrected according to age using the Von Betalanffy function. In the case of ultrasound data, for which the age of the population varied widely, it is necessary to develop a method to minimize variation by age before comparison with data obtained using other correction methods.

\section{Genetic correlation and selection response}

Comparison of the same traits in the carcass and ultrasound records (Table 5) showed that the genetic correlation of EMA and UEMA was approximately 0.41 , while that between BF and UBF was estimated to be 0.78 and that between MS and UMS was 0.60. Previous investigations estimating the genetic correlation between ultrasound and carcass traits were mainly focused on ultrasound measurements gathered within two months of slaughter and data from steers (Lee and Kim, 2004; Kim et al., 2012). Positive genetic correlations higher than values of 0.80 were observed for the same traits among all the traits from these studies. Nevertheless, the cattle evaluated and the data collection procedure adopted in the present study differed from those of previously conducted studies; therefore, these findings and those of previous

Table 5. Estimates of genetic parameters for carcass traits measured by ultrasound and observation of real carcasses with a multiple traits mixed animal model in Hanwoo cows

\begin{tabular}{lccccrrrr}
\hline & \multicolumn{3}{c}{ Ultrasound-scan measurement } & & \multicolumn{3}{c}{ Real Carcass measurement } \\
\cline { 2 - 4 } \cline { 7 - 9 } & UEMA & UBF & UMS & & EMA & BF & MS \\
\hline \hline UEMA & $\mathbf{0 . 3 3}$ & 0.42 & 0.47 & & $\mathbf{0 . 4 1}$ & 0.01 & 0.02 \\
UBF & 0.40 & $\mathbf{0 . 6 1}$ & 0.43 & & -0.52 & $\mathbf{0 . 7 8}$ & -0.07 \\
UMS & 0.30 & 0.21 & - & $\mathbf{0 . 4 6}$ & & -0.02 & 0.15 & $\mathbf{0 . 6 7}$ \\
EMA & - & - & - & - & & $\mathbf{0 . 2 9}$ & -0.64 & 0.24 \\
BF & - & - & - & - & & 0.36 & $\mathbf{0 . 4 0}$ & -0.06 \\
MS & - & - & - & & 0.21 & 0.20 & $\mathbf{0 . 3 8}$ \\
\hline
\end{tabular}

Heritabilities are on diagonals, genetic correlations are upper diagonals and phenotypic correlations are lower diagonals; Abbreviations: UEMA, ultrasound-scanned eye muscle area; UBF, ultrasound-scanned backfat thickness; UMS, ultrasound-scanned marbling score; EMA, eye muscle area on carcass; $\mathrm{BF}$, backfat thickness on carcass; MS, marbling score on carcass 
studies cannot be used for comparison. Crews et al. (2003) proposed estimated genetic correlations between ultrasound records and carcass records that were similar to those of the present study (EMA $0.54, \quad \mathrm{BF} 0.83$ and $\mathrm{MS}$ 0.69) in an investigation of Simmental heifers and steers. They reported that there is potential to improve genetic evaluation based on ultrasound traits and conventional carcass traits because high heritability and genetic correlation were found in BF, MS and EMA.

When the next generation is produced by individual selection through some traits, not only that trait, but also genetically related traits, are changed. In the present study, relatively high genetic correlation estimates between ultrasound traits and carcass traits were proposed. The correlation responses for the carcass traits based on corresponding ultrasound measurements were estimated to infer how great the genetic gains that could be accomplished would be (Table 6). Assuming $50 \%$ selection (selection intensity $=0.80$ ), the correlation response was estimated to be $0.97 \mathrm{~cm}^{2}$ for EMA, $-1.6 \mathrm{~mm}$ for and 0.4 for MS.

Table 6. Selection responses for carcass traits based on correlated corresponding traits measured by ultrasound-scan with an assumed selection intensity of 0.8 in Hanwoo cows

\begin{tabular}{lcccr}
\hline Traits & $h_{x}{ }^{1)}$ & ${r_{G}}^{2)}$ & $\sigma_{y}{ }^{3)}$ & $\triangle G_{y} /$ gen $^{4)}$ \\
\hline \hline EMA $\left(\mathrm{cm}^{2}\right)$ & 0.574 & 0.411 & 5.157 & 0.974 \\
BF (mm) & 0.781 & 0.779 & 0.779 & -1.604 \\
MS (score) & 0.678 & 0.673 & 1.147 & 0.420 \\
\hline
\end{tabular}

Abbreviations: EMA, eye muscle area; BF, backfat thickness; MS, marbling score;

${ }^{1)} h_{x}=$ estimated square root of heritability for corresponding traits measured by ultrasound scan;

2) $r_{G}=$ genetic correlation of traits between real and ultrasound scan measurements;

3) $\sigma_{y}=$ estimated genetic standard deviation of the trait;

4) $\triangle G_{y} /$ gen = genetic gain per generation;

\section{ACKNOWLEDGMENTS}

The study was carried out as a part of the "Study on the establishment of a breeding strategy for cows in the farm area and characteristics of genetic diversity for the manifestation of unusual coat color in Hanwoo" (Project no. PJ907087) supported by the Rural Development Administration, Republic of Korea.

\section{REFERENCES}

Cho, C. I. 2013. Accuracy of breeding value prediction and genetic gain of carcass traits in Hanwoo breeding population through application of genomic selection technology. Hankyong National University. Ph.D. Dissertation, Hankyong National University, Ansung, Korea.

Choi, T. J., Kim, S. D., Salces, Agapita J., Baik, D. H. 2006. Genetic parameter estimation on the growth and carcass trait in Hanwoo (Korean Cattle). J. Anim. Sci. \& Technol. (Kor.) 48:759-766.

Crews, D. H. and Kemp, R. A. 2001. Genetic parameters for ultrasound and carcass measures of yield and quality among replacement and slaughter beef cattle. J. Anim. Sci. 79:30083020 .

Crews, D. H., Pollak, E. J., Weaber, R. L., Quaas, R. L. and Lipsey, R. J. 2003. Genetic parameters for carcass traits and their live animal indicators in Simmental. J. Anim. Sci. 81: 1427-1433

Devitt, C. J. and Wilton, J. W. 2001. Genetic correlation estimates between ultrasound measurements on yearling bulls and carcass measurements on finished steers. J. Anim. Sci. 79:2790-2797.

Gilmour, A. R., Thompson, R. and Cullis, B. R. 1995. Average information REML: An efficient algorithm for variance parameter estimation in linear mixed models. Biometrics 51: 1440-1450.

Hirooka, H., Groen, A. F. and Matsumoto, M. 1996. Genetic parameters for growth and carcass traits in Japanese brown cattle estimated from field records. J. Anim. Sci. 74:21122116.

Kemp, D. J., Herring, W. O. and Kaiser, C. J. 2002. Genetic and environmental parameters for steer ultrasound and carcass traits. J. Anim. Sci. 80:1489-1496.

Koo, Y. M. 2007. Estimation of Genetic Correlation and Parameter for Ultrasound Measurements and Performance Test Data in Hanwoo Cows (Korean Cattle). MS. Dissertation, Gyeongsang National University, Chinju, Korea.

Kim, H. C., Lee, S. H., Dang, C. G., Jeon, G. J., Yeon, S. H., Cho, Y. M., Lee, S. M., Yang, B. S. and Kim, J. B. 2012. Estimation of Genetic Parameters for Ultrasound and Carcass Traits in Hanwoo. J. Anim. Sci. \& Technol. (Kor.) 54:331-336. Kim, H. S., Hwang, J. M., Choi, T. J., Park, B. H., Cho, K. H., Park, C. J. and Kim, S. D. 2010. Research on the reformation of the selection index for Hanwoo provan bull. J. Anim. Sci. \& Technol. (Kor.) 52:83-90.

Lee, D. H. 2003. Monte Carlo Simulations of Selection Responses 
for Improving High Meat Qualities Using Real Time

Ultrasound in Korean Cattle. J. Anim. Sci. \& Technol. (Kor.) 45:343-354.

Lee, D. H., Lee, G. H., Cho, C. I. and Kim, N. S. 2008. Effects of Body condition Score and Estimation of Growth Curves for Chest Girth and Ultra Sonic Longissimus Muscle Area, Backfat Thickness and Marbling Scores in Hanwoo (Korean cattle) Cows. J. Anim. Sci. \& Technol. (Kor.) 50:581-590.

Lee, J. H., Yeo, J. S. 2011. Estimation of genetic parameters using real-time ultrasound measurements in Hanwoo. Journal of the Korean Data \& Information Science Society. 22:1145-1152.

Lee, D. H., Choudhary, V. and Lee, G. H. 2006. Genetic Parameter Estimates for Ultrasonic Meat Qualities in Hanwoo Cows. Asian-Aust. J. Anim. Sci. 19:468-474.

Lee, D. H. and Kim, H. C. 2004. Genetic Relationship between Ultrasonic and Carcass Measurements for Meat Qualities in Korean Steers. Asian-Aust. J. Anim. Sci. 17:6-12.

Misztal, I., Tsuruta, S., Strabel, T., Auvray, B., Druet, T. and Lee, D. H. 2002. BLUPF90 and related programs (BGF90). Proc. 7th world congress genetics and applied livestock production, Montpellier, France. CD-ROM communication 28:07. Available via http://nce.ads.uga.edu/ ignacy/programs.html

National Institute of Animal Science. 2008. Report of genetic evaluation for Hanwoo breeding. Vol. 21:P12.

Park, B. H., Choi, T. J., Cho, K. H., Choi, J. G., Lee, S. S., Chung, H. Y., Kim, S. D., Kim, H. S., Hwang, J. M. and Lee, S. S. 2010. Comparison of Hanwoo proven and young bulls for major economic traits. J. Anim. Sci. \& Technol. (Kor.) 52:253-258.

Reverter, A., Johnston, D. J., Graser, H. U., Wolcott, M. L. and
Upton, W. H. 2000. Genetic analyses of live-animal ultrasound and abattoir carcass traits in Australian Angus and Hereford cattle. J. Anim. Sci. 78:1786-1795.

Roh, S. H. 2004. Comparison between REML and Gibbs Sampling algorithm with a mixed animal model to estimate genetic parameters for carcass traits in Hanwoo (Korean Native Cattle). MS. Dissertation, Gyeongsang National University, Chinju, Korea.

Roh, S. H., Kim, C. Y., Won, Y. S., Park, C. J., Lee, S. S. and Lee. J. G. 2010. Studies on genetic parameter estimation and sire selection to ultrasound measurement traits of Hanwoo. J. Anim. Sci. \& Technol. (Kor.) 52:1-8.

Stelzleni, A. M., Perkins, T. L., Brown, A. H., Pohlman, Jr., F. W., Johnson, Z. B. and Sandelin, B. A. 2002. Genetic parameter estimates of yearling live animal ultrasonic measurements in brangus cattle. J. Anim. Sci. 80:3150-3153.

Utrera, A. R. and Van Vleck, L. D. 2004. Heritability estimates for carcass traits of cattle: a review. Genetics and Molecular Research 3:380-394.

Von Bertalanffy, L. 1957. Quantitative laws in metabolism and growth. Q. Rev. Biol. 32:217-230.

Yoon, W. J. 2010. Study on Genetic Improvement for Meat Quality of Hanwoo Cows using Ultrasonic Measurements. Ph.D. Dissertation, Hankyong National University, Ansung, Korea. Yoon, H. B., Kim, S. D., Na, S. H., Chang, U. M., Lee, H. K., Jeon, G. J. and Lee, D. H. 2002. Estimation of genetic parameters for carcass traits in Hanwoo steer. J. Anim. Sci. \& Technol. (Kor.) 44:383-390.

(Received Sep. 11, 2013; Revised Nov. 20, 2013; Accepted Nov. 21, 2013) 